\title{
Levantamento do potencial de comercialização de produtos orgânicos para o estado de Mato Grosso do Sul
}

Evaluation of the potential market for organic products in the state of Mato Grosso do Sul (Brazil)

\author{
Levantamiento del potencial de comercialización de productos orgánicos para el \\ estado de Mato Grosso del Sur (Brasil) \\ Suppression du potentiel pour la commercialisation de produits biologiques pour l'état du Mato \\ Grosso do Sul
}

\author{
Cristiane Maria Vendramini Momesso* \\ Antonia Railda Roel ${ }^{* *}$ \\ Simone Palma Favaro ${ }^{* * *}$
}

Recebido em 20/9/2008; revisado e aprovado em 10/10/2008; aceito em 29/1/2009

\begin{abstract}
Resumo: Na avaliação sobre o potencial de mercado consumidor de produtos orgânicos em Campo Grande, MS, conclui-se que a maioria das mulheres entrevistadas afirmou conhecer o produto orgânico, porém, apenas aproximadamente a metade realmente sabe seu significado. Cerca de $52 \%$ delas pagaria mais caro por esse produto e estariam dispostas a procurar local próprio de comercialização (63,23\%). Após esclarecimentos sobre os benefícios do produto orgânico, quase a totalidade $(99,35 \%)$ optaria por estes produtos.

Palavras-chave: Desenvolvimento local. Agroecologia. Sustentabilidade. Segurança alimentar.
\end{abstract}

Abstract: Evaluation the potential market in Campo Grande for organic products, it was concluded that most of the interviewed women declared to know organic product is, although only a half part of them actually knew its meaning. Around $52 \%$ of the evaluated group would spend higher values to acquire organic products, and $66.23 \%$ were willing in going to a specific market place. Almost the entirely interviewed women (939.35\%) would choose organic products after an explanation about their benefits.

Keywords: Local development. Agroecology. Sustainability. Food assurance.

Résumé: Pour évaluer le potentiel du marché de consommation pour les produits biologiques à Campo Grande, MS, a conclu que la majorité des femmes interrogées ont déclaré connaître les produits biologiques, cependant, seulement environ la moitié sait vraiment son importance. Environ $52 \%$ d'entre eux paient plus cher pour ce produit et être prêts à chercher leur propre mise sur le marché $(63,23 \%)$. Après des éclaircissements sur les avantages des produits biologiques, la quasi-totalité $(99,35 \%)$ optent pour ces produits.

Mots-clés: Le développement local. Agroécologie. La durabilité. La sécurité alimentaire.

Resumen: La evaluación del potencial de mercado consumidor de productos orgánicos en Campo Grande, MS, he llegado a la conclusión de que la mayoría de las mujeres entrevistadas afirmaran conocer el producto ecológico, sin embargo, sólo aproximadamente la mitad sabe realmente su significado. Aproximadamente el $52 \%$ de ellas pagarían más caro por ese producto y estarían dispuestas a ir asta tiendas específicas para su comercialización (63,23\%). Después de una aclaración sobre los beneficios del producto ecológico, casi la totalidad $(99,35 \%)$ optarían por estos productos.

Palabras clave: Desarrollo local. Agroecología. La sostenibilidad. La seguridad alimentaria.

\section{Introdução}

A exposição a pesticidas agrícolas é frequentemente associada a riscos para a saúde humana, e atualmente com a promoção ao mal de Parkinson (XUE-FENG WANG et al., 2006). O meio ambiente também é fortemente impactado pelo uso contínuo dos pesticidas.
Estudo de impacto ambiental causado pelo uso de pesticidas agrícolas na Inglaterra mostrou uma redução nos índices avaliados e o autor atribuiu este comportamento uma exigência dos consumidores e reforça que esta tendência deveria ser acompanhada por políticas governamentais e nortear a produção industrial (CROSS e EDWARDS-JONES, 2006).

\footnotetext{
* Nutricionista - Mestre em Desenvolvimento Local. Universidade Católica Dom Bosco, UCDB, Campo Grande, MS. E-mail: momesso1@terra.com.br

** Engenheira Agrônoma, Dra. Entomologista pela Escola Superior de Agricultura "Luiz de Queiroz" ESALQ/ USP. Atualmente profa. Mestrado em Biotecnologia, UCDB, Campo Grande, MS. E-mail: arroel@ucdb.br *** Engenheira Agrônoma, Dra. Ciências dos Alimentos pela Universidade Estadual de Londrina UEL. Atualmente prof ${ }^{a}$ Mestrado em Biotecnologia, UCDB, Campo Grande, MS. E-mail: simone.palma@ucdb.br
} 
Recena et al. (2006) realizaram um levantamento epidemiológico no período de 1992 a 2002 no estado do Mato Grosso do Sul quanto às ocorrências de intoxicação registradas oficialmente. O estudo mostrou que a média de letalidade neste estado foi três vezes superior à média brasileira. A capital do estado, Campo Grande apresentou o índice mais alto no ano de 2000 , com 100,5 casos para cada 100.000 habitantes.

Em opção ao uso massivo de pesticidas a agricultura orgânica apresenta-se como um mecanismo de produção, baseado no conhecimento dos processos ecológicos, buscando mudanças socioeconômicas para a promoção da sustentabilidade de todos os setores do sistema alimentar (GLIESSMAN, 2001). A produção por mecanismos orgânicos além de oferecer alimentos inócuos tem mostrado superioridade na qualidade nutricional. Worthington (2001) realizou um estudo a partir da literatura existente sobre a comparação do produto orgânico e o convencional de frutas, vegetais e grãos e concluiu que o produto orgânico contém mais vitamina $C$, ferro, magnésio e fósforo; menos nitrato e menor teor de metais pesados que o produto convencional, porém não obteve resultados significantes quanto às proteínas.

De acordo com Soel (apud YUSSEF, 2004) mais de 24 milhões de hectares são administrados organicamente no mundo. A parte mais significativa desta área está localizada na Austrália (10 milhões de hectares). A agricultura orgânica é praticada em aproximadamente 100 países. A América Latina é a segunda em cultivo de terras orgânicas, estando a Argentina em primeiro lugar, seguido do Brasil com aproximadamente 841 mil hectares (YUSSEF, 2004). Em torno de $70 \%$ da produção brasileira, situa-se nos Estados do Paraná, São Paulo, Rio Grande do Sul, Minas Gerais e Espírito Santo. O crescimento nas vendas de orgânicos tem alcançado um ritmo de $50 \%$ ao ano (DAROLT, 2002). O Estado de Mato Grosso do Sul está iniciando a produção de orgânicos com alguns produtores em associações, mas com potencial considerável de crescimento.

A agricultura orgânica destaca-se pelo respeito ao meio ambiente e à humanidade, pois é tida como agricultura sustentável. Há considerável redução da dependência do comércio e da indústria. Sendo assim, está inserida no processo de desenvolvimento local, abrindo portas para o pequeno agricultor neste mundo globalizado. Segundo Ávila et al. (2000), considera que o Desenvolvimento Local não é um processo que usa em um primeiro momento reativar a economia e sim a cultura, a visão, o entendimento das pessoas, ou seja, o processo flui do sócio-cultural para o econômico. O Desenvolvimento Local é ativador da capacidade das pessoas a gerarem atividades, e daí criarem renda.

Órgãos governamentais envolvidos diretamente com a reforma agrária estudam formas de viabilizar a sustentabilidade das populações em assentamentos rurais, aldeias indígenas e pequenos produtores em geral, freqüentemente em regiões próximas a áreas de preservação. Cerca de três quartos da área do bioma Pantanal pertence ao estado de Mato Grosso do Sul e as práticas agrícolas podem constituir-se ou não em fontes de contaminação a esse ecossistema. Portanto, há uma oferta considerável de prováveis produtores rurais que buscam a alternativa mais viável rumo a agricultura sustentável, sendo assim nos encontramos diante da questão fundamental: há mercado potencial para os produtos orgânicos em Campo Grande?

Objetivou-se analisar o potencial de mercado consumidor do produto orgânico em Campo Grande, MS, maior população do estado. Pretendeu-se conhecer o mercado consumidor do produto orgânico; verificar se o público alvo está sensibilizado quanto à qualidade do produto; identificar se o comprador destes produtos irá a um mercado local específico e detectar se as pessoas eventualmente pagariam mais por estes produtos.

\section{Material e métodos}

A partir do censo demográfico de 2000 (IBGE), obteve-se o total de bairros existentes em Campo Grande e o total de mulheres residentes nestes bairros, optando-se por seis bairros com maior faixa de renda mensal, pois o produto orgânico ainda é utilizado por uma clientela diferenciada, devido ao elevado custo atual. O tamanho da amostra populacional foi obtido de acordo com Rea e Parker (1999), trabalhando com erro amostral de $10 \%$, totalizando 100 mulheres 
no mínimo Os dados foram obtidos por meio da aplicação de questionários totalizando 154 mulheres, no período de maio a setembro de 2005. As informações contidas nos questionários aplicados foram agrupadas por idade, escolaridade e renda familiar para se obter o perfil do atual consumidor e o potencial em Campo Grande, MS.

Em uma segunda investigação de demanda, foi realizada uma pesquisa nos três hipermercados existentes na cidade de Campo Grande, MS. Esta pesquisa aconteceu verbalmente, com o objetivo de conhecer os produtos cadastrados em cada loja e a demanda em relação aos mesmos.

Os dados coletados, resposta das pes- soas que participaram da entrevista, foram transformados em porcentagem inseridos em tabelas ou gráficos.

\section{Resultados e discussão}

A distribuição da faixa etária das pessoas que fizeram parte da pesquisa mostrou que a maioria possui idade acima de 21 anos, $40,91 \%$ possuem de 36 a 50 anos de idade, $27,92 \%$ de 21 a 35 anos, seguido de $25,97 \%$, acima de 50 anos. Sobre a escolaridade, a maioria delas possui o superior completo, com $55,19 \%$, seguido de $16,23 \%$ com segundo grau completo e $14,29 \%$ com superior incompleto (Tabela 1).

Tabela 1 - Perfil da População Amostrada (154 Mulheres), para o levantamento do Mercado Consumidor Potencial de Produtos Orgânicos no Município de Campo Grande, MS.

\begin{tabular}{cccccc}
\hline \multicolumn{2}{c}{ Faixa etária } & \multicolumn{2}{c}{ Grau de instrução } & \multicolumn{2}{c}{ Renda familiar } \\
\hline $\begin{array}{c}\text { Proporção } \\
(\%)\end{array}$ & $\begin{array}{c}\text { Idade } \\
(\text { anos })\end{array}$ & $\begin{array}{c}\text { Proporção } \\
(\%)\end{array}$ & Escolaridade & $\begin{array}{c}\text { Proporção } \\
(\%)\end{array}$ & $\begin{array}{c}\text { Salários } \\
\text { mínimos }\end{array}$ \\
\hline 3,90 & até 20 & 3,90 & $1^{\circ}$ grau incompleto & 5,19 & até 2 \\
27,92 & 21 a 35 & 5,19 & $1^{\circ}$ grau completo & 16,23 & 3 a 5 \\
40,91 & 36 a 50 & 4,55 & $2^{\circ}$ grau incompleto & 11,69 & 6 a 8 \\
25,97 & $>$ de 50 & 16,23 & $2^{\circ}$ grau completo & 14,94 & 9 a 11 \\
1,30 & sem resposta & 14,29 & superior incompleto & 50 & $>$ de 12 \\
- & - & 55,19 & superior completo & - & - \\
- & - & 0,65 & sem resposta & - & - \\
\hline
\end{tabular}

Sobre a renda familiar $50 \%$ das mulheres questionadas referem-se ao recebimento de mais de 12 salários mínimos mensais, resultado semelhante observado por Ruchinski et al. (1999) sobre o perfil dos consumidores de orgânicos nas feiras verdes no município de Curitiba.
Apesar de Campo Grande estar iniciando a comercialização, 55,19\% destes afirmaram que consomem produtos orgânicos às vezes, $14,94 \%$ "sempre consomem", $18,83 \%$ não responderam e 11,04\% "nunca consomem" (Gráfico 1). As pessoas que conhecem orgânicos, nunca consomem por "não achar o produto". 


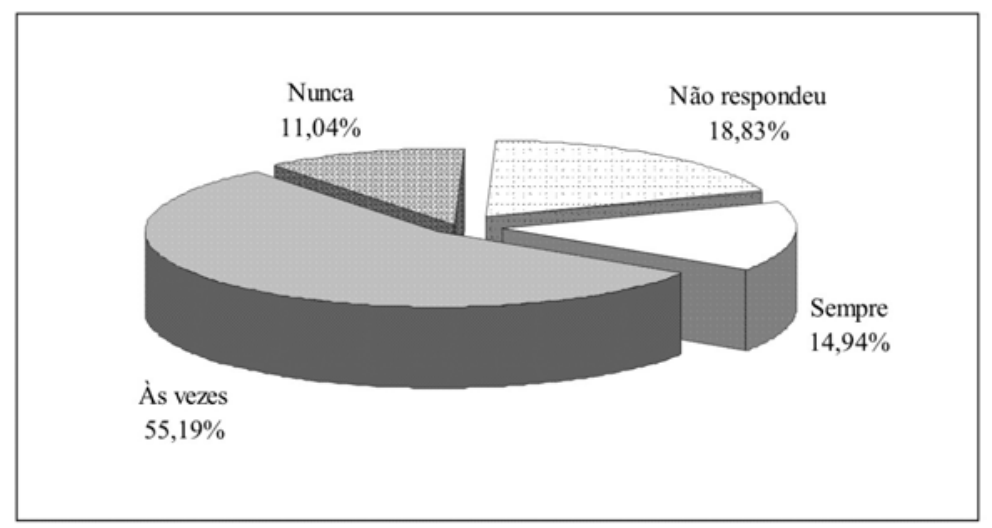

Gráfico 1 -Freqüência de consumo de produtos orgânicos dos entrevistados sobre potencial de consumidores de produtos orgânicos em Campo Grande, MS, 2005.

Para identificar se realmente sabem o que significa o produto orgânico, foi solicitada a descrição deste. Das pessoas que afirmaram saber, a maioria $(54,40 \%)$ respondeu corretamente, limitando-se a responder que "o produto orgânico é um produto sem agrotóxico ou sem produtos químicos". Das demais, 16,0\% não sabiam responder, inclusive parte delas respondeu que "eram produtos cultivados na água", enquanto $29,6 \%$ não responderam, o que denuncia que não sabiam seu significado (Gráfico 3).

Ficou evidente que as pessoas confundem "produtos orgânicos" com "integrais", "naturais" e "hidropônicos". Nos supermercados esses produtos são colocados próximos aos orgânicos, o que pode provocar tal equívoco. A falta de informação leva os consumidores preocupados com a saúde, a adquirir hidropônico, que são cultivados com insumos agrícolas sintéticos. Em hidroponia, as soluções usadas são ricas em nitrato, na forma prontamente disponível e em condições favoráveis à absorção pelas raízes. Assim, os teores de nitrato nos produtos hidropônicos tendem a ser superiores aos observados nas plantas cultivadas em outros sistemas (FAQUIN e ANDRADE, 2004). Em Florianópolis $94,50 \%$ dos entrevistados responderam corretamente o significado de produto orgânico, maior que a encontrada na presente investigação (KOHLRAUSCH et al., 2004). A autora ainda relata que naquela cidade há informações em supermercados e na mídia, o que não ocorre em Campo Grande.

Quando o assunto é preço, 37,01\% das pessoas que participaram da avaliação não responderam à questão. Apenas $24,03 \%$ pagariam $10 \%$ a mais pelo produto, $22,73 \%$ pagariam $5 \%$ a mais, $9,74 \%$ pagariam $20 \%$ ou mais e $6,49 \%$ pagariam $15 \%$ a mais (Gráfico 2). O produto orgânico apresenta freqüentemente preço superior ao produto convencional, no entanto não é necessariamente mais caro, pois não há utilização de produtos sintéticos que encarecem a produção, por outro lado há maior utilização de mão de obra e perdas ocasionais. Há ainda a questão da demanda maior que a oferta e disposição de consumidores em pagar pelo produto.



Gráfico 2 -Porcentagem excedente que os entrevistados sobre potencial de consumidores de produtos orgânicos em Campo Grande, MS, 2005, pagariam por estes produtos. 
A afirmação de que o "consumidor em potencial pagaria mais caro pelo produto diferenciado" é semelhante a pesquisas realizadas em outros estados, como a do Paraná feita por Darolt (2002), que, apesar da maioria $(62,7 \%)$ afirmar que considera os preços mais caros em relação aos convencionais, o consumo continua crescendo.

Altieri e Mazera (1998) demonstraram que os métodos são inadequados para uma análise mais ampla, nos quais é dada dimen- são econômica sem abordar análises em longo prazo, como mudanças ambientais, perdas de solo, de recursos genéticos além de reflexos sociais e da qualidade dos alimentos.

Sobre a comercialização, $81,17 \%$ afirmam conhecer o produto orgânico (Tabela 2 ), mas apenas $54,40 \%$ realmente conhecem (Gráfico 3). A falta de informação é um problema atualmente encontrado para comercialização desses no estado e campanhas de divulgação são necessárias.

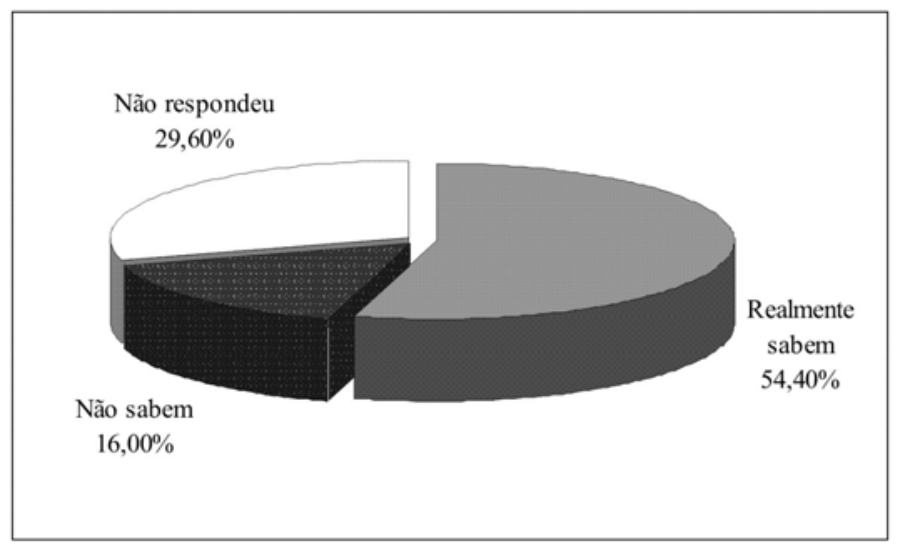

Gráfico 3 -Respostas descritivas das 125 mulheres que afirmaram que sabem o que significa o produto orgânico no questionário aplicado sobre potencial de consumidores de produtos orgânicos em Campo Grande, MS, 2005.

Quase a metade dos entrevistados diz procurar por produtos orgânicos $(44,80 \%)$. A maioria destes está disposta a pagar mais caro por estes produtos, $51,95 \%$ dos entrevistados, os quais $5 \%$, a $20 \%$ a mais que o dos produtos convencionais (Tabela 2).

Tabela 2 - Respostas afirmativas e negativas, referentes à comercialização de produtos orgânicos, dos entrevistados sobre o potencial de consumidores destes alimentos em Campo Grande, MS, 2005.

\begin{tabular}{lccc}
\hline Questões & Sim & Não & Não respondeu \\
\hline Sabe o que significa produto orgânico? & $81,17 \%$ & $18,83 \%$ & - \\
Procura por produtos orgânicos? & $44,80 \%$ & $33,77 \%$ & $21,43 \%$ \\
Pagaria mais caro por este produto orgânico? & $51,95 \%$ & $27,27 \%$ & $20,78 \%$ \\
Iria a um local específico de venda? & $63,23 \%$ & $13,64 \%$ & $20,13 \%$ \\
\hline
\end{tabular}

Sobre o local de comercialização, $63,23 \%$ das mulheres estão dispostas a se deslocarem ao local específico, desde que encontrem variedade de produtos em local acessível. A comercialização em algumas cidades é feita em locais próprios e vendas diretamente do produtor.
Após a explicação sobre as vantagens e qualidades do produto orgânico aos entrevistados, foram novamente questionados: "Você agora, compraria produtos orgânicos?" Como resposta 99,35\% das pessoas afirmou que "sim" (Tabela 3). 
Tabela 3 - Comparação entre as respostas afirmativas de compra do produto orgânico antes e após a sensibilização, na qual se explica resumidamente o que significa e as vantagens do produto orgânico em Campo Grande, MS, 2005.

\begin{tabular}{lcc}
\hline Questões & Antes da sensibilização & Após sensibilização \\
\hline Comprariam produtos orgânicos & $71,43 \%$ & $99,35 \%$ \\
Não comprariam produtos orgânicos & $1,30 \%$ & $0,65 \%$ \\
Comprariam dependendo do preço & $14,93 \%$ & - \\
Comprariam dependendo da facilidade & $9,74 \%$ & - \\
Não responderam & $2,60 \%$ & - \\
\hline
\end{tabular}

Confrontando os dados obtidos a partir das mulheres que realmente sabem o que significa o produto orgânico e os consomem, obteve-se uma amostragem do perfil do consumidor atual de Campo Grande, MS. Em relação à freqüência de consumo apenas $11,76 \%$ afirmam consumir com freqüência e $70,59 \%$ às vezes, $14,71 \%$ dizem nunca consumir produtos orgânicos e 2,94\% não responderam (Gráfico 4).

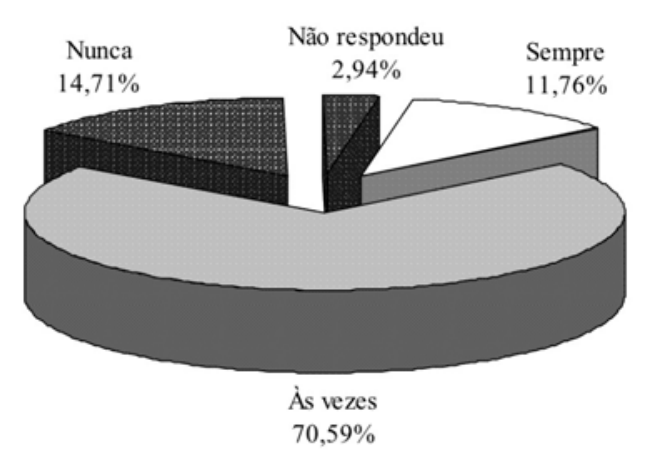

Gráfico 4 -Freqüência de consumo de produtos orgânicos dos entrevistados sobre potencial de consumidores de produtos orgânicos em Campo Grande, MS, 2005, que realmente conhecem o que significa o produto orgânico.

Estabeleceu-se o perfil do real consumidor deste tipo de produto em Campo Grande, MS (Tabela 4). Constatou-se que as pessoas que conhecem o produto e o consomem, enquadram-se na faixa etária de 36 a 50 anos, possuem grau superior completo e renda superior a 12 salários mínimos. De acor- do com Darolt (2002) existem dois tipos de consumidores: os mais antigos, motivados e bem informados e exigentes em termos de qualidade biológica do produto, e os mais recentes de pouca escolaridade, freqüentadores das grandes redes de supermercados.

Tabela 4 - Perfil do Consumidor de Produtos Orgânicos no Município de Campo Grande, MS, 2005.

\begin{tabular}{ccclcc}
\hline \multicolumn{2}{c}{ Faixa etária } & \multicolumn{2}{c}{ Grau de instrução } & \multicolumn{2}{c}{ Renda familiar } \\
\hline $\begin{array}{c}\text { Proporção } \\
(\%)\end{array}$ & Idade & $\begin{array}{c}\text { Proporção } \\
(\text { anos })\end{array}$ & $\begin{array}{c}\text { Escolaridade } \\
\text { (\%) }\end{array}$ & $\begin{array}{c}\text { Proporção } \\
(\%)\end{array}$ & $\begin{array}{c}\text { Salários } \\
\text { mínimos }\end{array}$ \\
\hline 25 & 21 a 35 & 3,57 & $1^{\circ}$ grau incompleto & 1,79 & $<$ de 2 \\
25 & 36 a 50 & 19,64 & $2^{\circ}$ grau completo & 7,14 & 3 a 5 \\
21,42 & $>$ de 51 & 1,79 & $2^{\circ}$ grau incompleto & 7,14 & 6 a 8 \\
1,79 & sem resposta & 8,93 & superior incompleto & 14,28 & 9 a 11 \\
- & - & 64,28 & Superior completo & 67,86 & $>$ de 12 \\
\hline
\end{tabular}


Uma pesquisa encomendada pelo SEBRAE-PR realizada pelo Datacenso (2002) nos Estados do Sul e Sudeste mostrou que $53 \%$ das pessoas entrevistadas responderam que possuem o hábito de consumir alimentos orgânicos, principalmente da classe A (60\%). Ruchinski e Brandenburg (1999) após pesquisa em duas feiras verdes no município de Curitiba descreveram o perfil do consumidor orgânico: profissional liberal, na maioria do sexo feminino (66\%), idade entre 31 e 50 anos (62\% dos casos), nível de instrução elevada, maioria com curso superior. Declaram que conhecem os males dos agrotóxicos, tem ainda o hábito de praticar esporte $(54,9 \%)$ e $62,9 \%$ procuram estar em contato com a natureza $(62,9 \%)$. Os mesmos autores confrontaram a escolaridade de freqüentadores de feiras tradicionais e orgânicas e constataram que os últimos têm maior escolaridade (maioria curso universitário) e os primeiros, primeiro grau, sendo que destes somente 2,7\% sabem da existência de feiras orgânicas. A maior parte dos consumidores das feiras convencionais tem renda de até dez salários mínimos, e os de feira orgânica, $68 \%$ tem renda superior a nove salários mínimos.

Estudos efetuados na Itália, Inglaterra, França e Alemanha por Sylvander (1998), mostraram as razões para o baixo consumo de produtos orgânico pela população, em primeiro lugar está o preço, seguido da oferta insuficiente e ainda a dúvida sobre a procedência. A pesquisa mostrou que $15 \%$ dos consumidores estão sensibilizados a respeito dos alimentos orgânicos e que as informações para os consumidores ainda são insuficientes em $72 \%$ dos casos.

Apesar das dificuldades nas pesquisas nos três hipermercados da cidade, obtiveramse algumas informações. No setor de mercearia o gerente afirmou ter apenas café orgânico, porém verificou-se que havia ainda duas marcas de açúcar. De acordo com as respostas dos funcionários ficou a dúvida se estes sabem o que significa produtos orgânicos? No setor de frutas, verduras e legumes há grande variedade de produtos, como: sucos prontos, rúcula, almeirão, cenoura, batata, espinafre, chuchu, abobrinha, e muitas frutas, como laranja, morango, banana prata e nanica. Todos os produtos com certificado do IBD ou Ecocert, entidades de certifi- cação conceituadas no Brasil. “As vendas destes produtos estão crescendo muito", conforme informou o gerente do setor; justificando o aumento da variedade dos orgânicos.

No segundo hipermercado, foram entrevistadas três pessoas, que não souberam informar sobre o produto, e deixaram evidente que confundem produtos orgânicos com naturais e integrais. A responsável pelos produtos naturais, relatou que vendeu orgânicos, feijão, soja, arroz, milho de pipoca e açúcar, por onze meses, e cessaram os pedidos por não haver saída. No terceiro hipermercado o gerente do setor da mercearia relatou que há demanda e que pretendem aumentar a variedade destes produtos.

Observa-se, portanto, que mesmo os gerentes de grandes centros de venda desconhecem o que significa produto orgânico. De maneira geral não há informação no local sobre estes produtos. O desconhecimento não só do consumidor como dos funcionários responsáveis pelas vendas e confundem com outros como "naturais, light, integrais e hidropônicos", muitas vezes colocados estrategicamente lado a lado e embalados de forma similar, também relatado também por Darolt (2002).

\section{Considerações finais}

Pequenos produtores, em especial assentados, na busca da sustentabilidade econômica de suas propriedades, vêem diante de uma questão fundamental "há mercado para produtos orgânicos?". Este questionamento evidencia a necessidade de um levantamento de dados que responda a esta preocupação.

Sendo assim, analisando os dados obtidos nesse levantamento de potencial da comercialização de produtos orgânicos na população feminina de Campo Grande, MS, em que: (1) Das 125 mulheres que afirmam saber o que significa o produto orgânico, $54,40 \%$ realmente sabem o que significa este produto ou pelo menos já ouviu falar, porém as demais confundem alimentos orgânicos com naturais, hidropônicos e outros; (2) A maioria da população amostrada está disposta a pagar até $15 \%$ a mais para o produto orgânico em relação ao convencional e a deslocar-se até um local específico de ven- 
da; (3) O perfil do consumidor feminino atual em Campo Grande, MS encontra-se entre a faixa etária de 36 a 50 anos, com grau de instrução superior completo e renda familiar acima de 12 salários mínimos (4) Após conhecimento do produto, a população avaliada consumiria produtos orgânicos.

Os dados evidenciam a necessidade de campanhas de esclarecimento sobre o que significa alimentos orgânicos e seus benefícios. Pois a população anseia por alimentos "limpos" e está disposta a consumi-lo e pagar por ele, garantindo assim, a comercialização. Após esse procedimento, produtores rurais que querem adotar os métodos orgânicos de produção de alimentos, podem se dedicar a essa atividade para comercialização em Campo Grande, MS. Desta maneira, assentados rurais, comunidades indígenas e demais pequenos agricultores poderiam alcançar a sustentabilidade econômica inserida em uma agricultura que por definição é ambientalmente sustentável e socialmente justa. Contribuindo assim para o que ocorra o desenvolvimento local.

\section{Referências}

ALTIERI, M.A.; MAZERA, O. Desenvolvimento rural sustentável na América Latina: construindo de baixo para cima. In: ALMEIDA, J.; NAVARRO, J. (Org.). Reconstruindo a agricultura: idéias e ideais na perspectiva do desenvolvimento rural sustentável. Porto Alegre: Editora da Universidade, 1998.

ÁVILA, V.F. de. Formação educacional em desenvolvimento local: relato de estudo em grupo e análise de conceitos. Campo Grande: UCDB, 2000.

CROSS, P.; EDWARDS-JONES, G. (in press). Variation in pesticide hazard from arable crop production in Great Britain from 1992 to 2002: pesticide risk indices and policy analysis, Crop Protection, v. 25, Issue, 10, October, 2006, p. 1101-1108.
DAROLT, M.R. A agricultura orgânica na América Latina. 2002. Disponível em: <http:// www.planetaorganico.com.br/trabdaroltal.htm>. Acesso em: 15 maio 2004.

DATACENSO. Mercado de produtos orgânicos: consumidor. Curitiba: SEBRAE, 2002. 89p.

FAQUIN, V.; ANDRADE, A.T. Nutrição mineral e diagnose do estado nutricional de hortaliças. Lavras: UFLA/FAEPE, 2004. 88 p.

GLIESSMAN, S.R. Agroecologia: processos ecológicos em agricultura sustentável. Stphen R. Gliessman. 2.ed. Porto Alegre: Editora da Universidade/UFRGS, 2001.

IBGE. Instituto Brasileiro de Geografia e Estatística. Unidade de MS. Setor de Divulgação. População dos bairros de Campo Grande. Censo Demográfico, 2000.

KOHLRAUSCH, A.K.; CAMPOS, L.M.S.; SELIG, P.M.O comportamento do consumidor de produtos orgânicos em Florianópolis: uma abordagem estratégica. Curitiba: Enampad, GAG250, 2004.

REA, L.; PARKER, R. Metodologia da pesquisa: do planejamento à execução. São Paulo: Pioneira, 1999. p. 129.

RECENA, M.C.P. et al. Acute poisoning with pesticides in the state of Mato Grosso do Sul, Brazil. Science of The Total Environment, v. 357, Issues 1-3, p. 88-95, 15 March 2006.

RUCINSKI, J.; BRANDENBURG, A. Organizações verdes: a relação produtor-consumidor de alimentos orgânicos. Relatório de projeto de pesquisa. Curitiba: Universidade Federal do Paraná, 1999.

SYLVANDER, B. Lê marche dês produits biologiques et al demande. Lê Mans: INRA-UREQUA, 1998. 27 p.

XUE-FENG W. et al. Inhibitory effects of pesticides on proteasome activity: Implication in Parkinson's disease. Neurobiology of Disease, v. 23, Issue 1, July 2006, p.198-205.

WORTHINGTON, M.S., Sc.D., C.N.S. Nutricional Quality of Organic Versus Conventional Fruits, Vegetables, and Grains. In: The Journal of alternative and complementary medicine, v. 7, n. 2, 2001, p. 167-173. Marry Ann Liebert, Inc.

YUSSEF, M. Development and State of organic agriculture worldwide. In: The world of organic agriculture - statistic and emerging trends. IFOAM, 2004. 